\title{
O cão como aspecto motivador de crianças com transtorno do espectro autismo
}

\section{The dog as a motivating aspect of children with spectrum autism disorder}

\author{
Maria Teresa Duarte Nogueira, Márcia de Oliveira Nobre, Rita de Cássia Morem Cóssio Rodriguez, Ana Laura Sica \\ Cruzeiro Szortyka, Fernanda Krug, Erika Scheidt Görgen, Anna Raffaella Borges Kramer, Vanessa de Gusmão \\ Santos,Juliana Corrêa Hertzberg, Tais Severo de Severo, Stephanie Duarte Bresolin, Rafaella Masseron Laviaguerre da \\ Silva, Josimara Gonçalves Schuster, Camila do Canto Perez, Isabella Maciel Heemann, Flora Beatriz Proiette Santos \\ Universidade Federal de Pelotas
}

\begin{abstract}
Resumen
Crianças com Transtorno do Espectro do Autismo (TEA) apresentam dificuldades na comunicação e interação social e padrões de comportamentos repetitivos e restritos. Com objetivo de verificar se a presença do cão na sessão terapêutica com crianças com TEA seria um aspecto motivador, foi realizado um estudo com 24 crianças na faixa etária de três a dez anos. Os resultados obtidos neste estudo foram tabulados e analisados de maneira inferencial, e a diferença entre as médias do tempo de reação foi utilizado o teste Mann Whitney. As diferenças significativas foram comprovadas através de um p-valor $\leq 0,005$. Este estudo demonstrou que as crianças do grupo controle apresentam uma média maior de tempo de reação à sessão em relação às do grupo experimental.

Palabras clave: motivação, terapia assistida por animais, transtorno do espectro do autismo
\end{abstract}

\begin{abstract}
Children with Autism Spectrum Disorder (ASD) present difficulties in communication and social interaction and patterns of repetitive and restricted behaviors. In order to verify if the presence of the dog in the therapeutic session with children with ASD would be a motivating aspect, a study was carried out with 24 children in the age group of three to ten years. The results obtained in this study were tabulated and analyzed in an inferential way, and the difference between the means of the reaction time was used the Mann Whitney test. The significant differences were verified through a $\mathrm{p}$-value $\leq 0.005$. This study demonstrated that the children in the control group had a higher average reaction time to the session compared to the experimental group.
\end{abstract}

Keywords: motivation, animal-assisted therapy, autism spectrum disorder

\section{Introdução}

Segundo a Delta Society, o trabalho terapêutico com animais pode ser definido em dois programas distintos a Atividade Assistida por Animais (AAA) e Terapia Assistida por Animais (TAA) (Capote, Costa, 2011).

Já a organização americana Pet Partners nos tráz um novo termo: as Intervenções Assistidas por Animais (IAA) que tem por missão promover melhorias em saúde. Classifica as IAA em três grandes categorías: a Terapia Assistida por Animais (TAA) abrange as intervenções terapêuticas que consiste na utilização da relação humano-animal como parte integrante do proceso terapéutico. É uma intervenção direcionada, com critérios específicos, objetivos claros e é realizada por profissional da área da saúde, focada no desenvolvimento e melhora de aspectos sociais, físicos, emocionais e cognitivos do indivíduo envolvido. A Atividade Assistida por Animais é o desenvolvimento de atividades de entretenimento, recreação, motivação e melhora da qualidade de vida e a Educação Assistida por Animais (EAA) são intervenções assistidas de cunho pedagógico (Chelini, 2016).

A observação da influência positiva dos animais com pessoas foi documentada pela primeira vez na Inglaterra quando pacientes foram incentivados a escrever, ler e se vestir com o auxilio de animais domésticos, além de programas de caridade que também salientavam que a presença de animais em hospitais para transtornos mentais promoviam uma atmosfera mais leve. Nos Estados Unidos, em 1944, cães foram inseridos terapeuticamente no hospital da Força Aérea Convalescente para estimular o bem estar dos internos (Dotti, 2005).

Outro importante documento relatando um aintervenção terapêutica com animais foi a de Boris Levinson. $\mathrm{O}$ psiquiatra tratando um menino de nove anos de idade concluiu que a presença do cão como co-terapeuta estabeleceu um clima de confiança e ajudou a desenvolver um sólido relacionamento com a criança (Eggiman, 2006).

No Brasil a partir de 1955 , os trabalhos da Dra. Nise da Silveira no hospital psiquiátrico Dom Pedro II, em Engenho de Dentro, no Rio de Janeiro, constituem parte das primeiras tentativas nacionais do uso dos animais com fins terapêuticos. Entretanto, décadas antes de se pensar em reforma psiquiátrica (Dotti, 2005).

A doutora Nise já acreditava na possibilidade de o sofrimento psíquico ser passível de expressão simbólica. Ela criou ateliês de pintura e modelagem, onde permitia que gatos e cachorros circulassem entre os pacientes. A partir de uma vivência da doutora, quando encontrou uma cadela abandonada no hospital e entregou-a aos cuidados de um doente, este assumiu o cuidado ao animalzinho e esta atitude fez com que este doente reatasse os vínculos com a realidade. "Isto fez com que 
ela percebesse que os animais possuíam qualidades que os tornavam um ponto de referência estável na vida do paciente, e em seu projeto atribui-lhes a função de coterapeutas" (p.15, Capote; Costa, 2011).

No que tange a estudos com pessoas autistas, existem várias pesquisas realizadas em outros países. Um estudo citado por Muñoz e Roma (2016) desenvolvido por Redefer e Goodman (1989) objetivou descobrir se um cão seria um auxiliar útil em sessões com crianças autistas e este estudo demonstrou que a Terapia Facilitada por cães poderia ser usada para ajudá-las a interagir com outras pessoas.

Vários autores descrevem sobre os efeitos positivos para os diferentes tipos de pacientes submetidos à Terapia Assistida por Animais. Um estudo com crianças em um ambiente de sala de aula e constatou que os animais proporcionam um sistema de apoio social e emocional, elevam a autoestima da criança e fazem com que seja mais fácil para a criança se expresar (Friesen, 2010).

Dotti (2005), relata sobre um estudo realizado com crianças autistas em 1999 a U.S. PetIndustry's Foundatione a Pet Care Trust lançaram um estudo para quantificar e compreender os efeitos da TAA com cães, comparando outros tipos de terapias tradicionais e os resultados indicaram que as crianças pareciam mais receptivas brincando com os cães, mais atentas e sorrindo mais na presença desses cães. Parece que, quando estão com os cães, mostram um nível maior de atividade, focada no interesse pelo ambiente.

Um outro estudo conduzido por François Martin e sua equipe na Universidade Estadual de Washington, citado por Dotti, (2005) demonstrou que os cães podem chamar a atenção das crianças autistas, pois constataram que as crianças olhavam para o cão e conversavam com ele por maior período de tempo do que com o terapeuta.

De acordo com Nobre, et al (2017) a Faculdade de Veterinária da Universidade Federal de Pelotas-RSBrasil, desde 2006 desenvolve um projeto de extensão Pet Terapia que realiza atividade, terapia e educação assistida por animais em diversas instituições do município de Pelotas (RS) e região. Conta aindacom uma equipe multidisciplinar composta por docentes, discentes da graduação e pós-graduação, profissionais da área da saúde e educação. As intervenções Assistidas por Animais (IAA), realizadas pelo Pet Terapia, abrangem Atividade (AAA), Educação (EAA) e Terapia Assistida por Animais (TAA) que são desenvolvidas de forma inter, multi e transdisciplinar com a equipe do projeto e das instituições assistidas, desde o planejamento, execução e avaliação.

Salienta Rosa, Rainho e Pereira (2015) sobre a importância dos terapeutas estarem atentos aos indicadores de bem-estar do cão e com capacidade de reconhecer e dar respostas a sinais de estresse, desconforto, medo que possam decorrer das terapias.

Seguindo as ideias de Nobre, et al (2017) no atendimento a crianças autistas o cão se mostra como um potencial elo entre a criança e o terapeuta. Assim, observa-seque aos poucos as crianças interagem com a equipe, desenvolvendo um vínculo afetivo com os cães, tornando perceptível a satisfação de ambos.
Partindo das ideias dos autores aqui apresentados, de que a Terapia Assistida por Animais é um tipo de intervenção em que o animal é parte integrante do processo do tratamento, promovendo uma melhora da função física, social, emocional e cognitiva. Além disso, que através deste método de terapia poderão ser trabalhadas as áreas relacionadas ao desenvolvimento psicomotor, desenvolvimento sensorial, distúrbios físicos e emocionais e algumas das principais características do (TEA) é o isolamento do mundo e a incapacidade de formar relações com o mundo exterior, entende-se que a inserção do cão como co-terapeuta poderá ajudar no estado físico e psicológico da criança com TEA.

\section{Método}

Com objetivo de de verificar se a presença do cão na sessão terapêutica com crianças com TEA seria um aspecto motivador, foi realizado um estudo com 24 crianças na faixa etária de três a dez anos atendidas pelo Centro de Atendimentoao Autista Dr. Danilo Rolim de Moura- Pelotas/RS- Brasil. Estas foram divididas em dois grupos de diagnóstico comparável. Um grupo chamado de grupo experimental, onde foram trabalhadas com a inserção do cão e o outro chamado grupo de controle trabalhado sem a inserção do cão. Foram realizadas 7 sessões individuais de TAA com dois grupos (experimental e controle). Os resultados obtidos neste estudo foram tabulados e analisados de maneira inferencial, e a diferença entre as médias do tempo de reação foi utilizado o teste Mann Whitney. As diferenças significativas foram comprovadas através de um p-valor $\leq 0,005$.

\section{Resultados e Discussões}

Este estudo demonstrou alguns resultados, apresentados na figura 1, significativos de análise referente ao tempo de reação, ou seja,é o tempo que a criança manifesta para reagir diante de uma sessão de psicoterapia. O teste Mann Whitney evidenciou diferenças significativas entre as médias de tempo de reação entre os dois grupos, As Crianças do grupo controle apresentaram uma média maior de tempo de reação à sessão em relação às crianças do grupo experimental $(p \leq 0,005)$. Segundo Muños e Roma (2016), existem pacientes autistas que são atraídos pelos cães e de imediato interagem, para outros o cão deva ser introduzido várias vezes na sessão até que a criança estabeleça uma interação com o animal. 


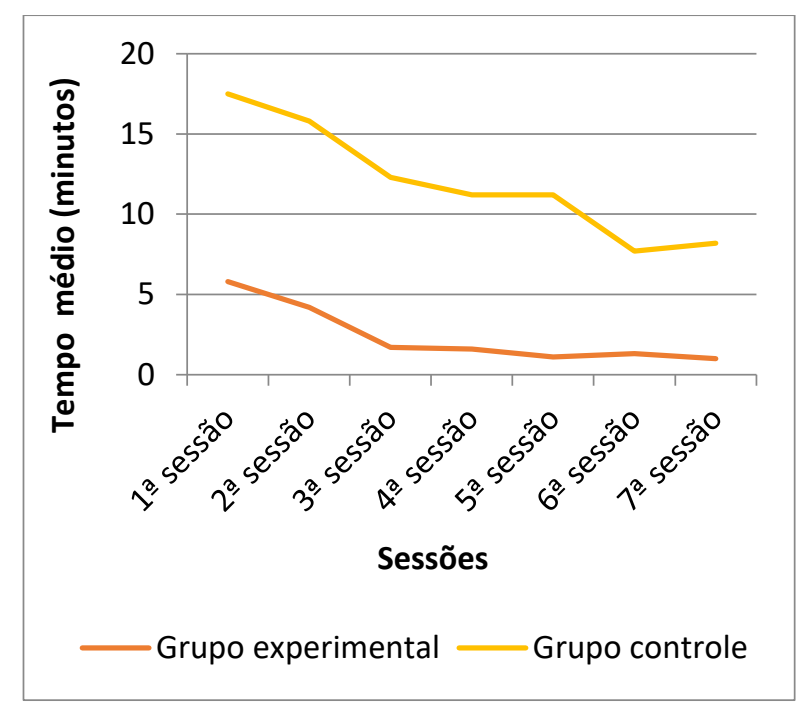

Figura 1: Demonstração do tempo de reação (média) de crianças comTranstorno do Espectro Autistas nas sessões de terapia com grupo experimental) e sem (grupo controle) a presença do cão como mediador no Centro de Atendimentoao Autista Dr. Danilo Rolim de Moura Pelotas-RS

Dentro deste contexto, podemos supor que a presença do animal contribuiu para uma imediata interação à TAA. De acordo com Friedmann, Katcher, Lynch, Messent, (1983); Myers, (1999) apud Dotti (2005), os animais são considerados de grande ajuda em uma psicoterapia, serão a ponte com o terapeuta e assim este poder alcançar mais rapidamente o paciente.

Para Muños e Roma (2016) a presença de um animal na terapia comcrianças autista pode oferecernovo foco de atenção, possibilitando a modulação da ansiedade e a abertura da possibilidade de vinculação entre paciente e terapeuta, poisassim como os animais, o autista percebe o mundo em termos sensoriais, o que poderia facilitar a interação.

Ainda no decorrer desse estudo, verificou-se que o desenvolvimento e avaliação da ação da TAA com crianças que apresentam o TEA aumentou a interação social através do aumento de regras sociais como a saudação e a despedida, a capacidade de imitação de gestos, a reciprocidade emocional, a expressão de sentimentos e emoções. Além de outros aspectos, como a motricidade global, quando comparadas ao uso do método de psicoterapia sem o cão. Os resultados do estudo da TAA evidenciaram sua importância para fins de intervenção social em crianças que apresentam o TEA demonstrando que o uso desta abordagem terapêutica amplia os comportamentos sociais, tais comointeresse, atenção e motivação conforme figura 2.

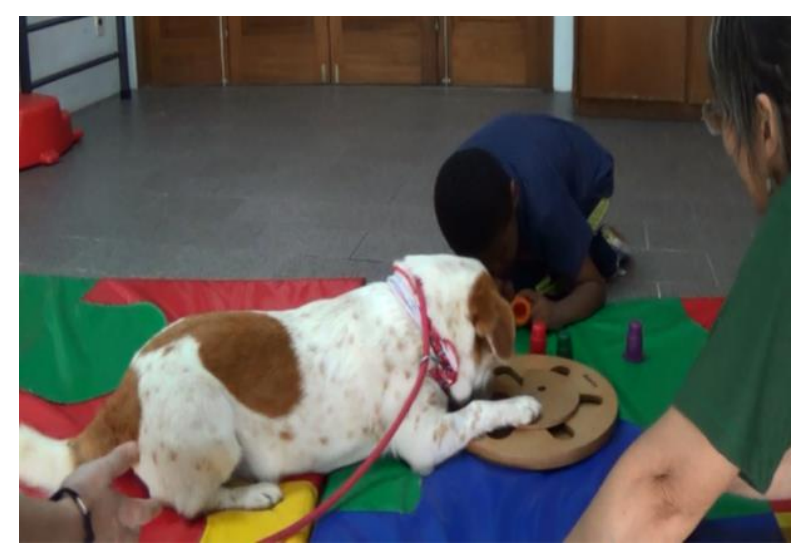

Figura 2: criança que apresenta TEA na TAA.

Neste estudo, verificou-se que o cão no desempenho do papel de co-terapeuta, facilitou a socialização e afetividade através do contato direto entre humano-animal; promoveu o desenvolvimento de vínculos e estimulou a expressão de emoções, ilustrado na figura 3 ,assim como provocou avanços em outras dimensões como a coordenação motora, a estímulação da memória, a comunicação, segurança, confiança, socialização, motivação, coordenação mototora ampla e fina, diminuição da ansiedade e ainda desenvolvimento de sentimentos de compaixão.

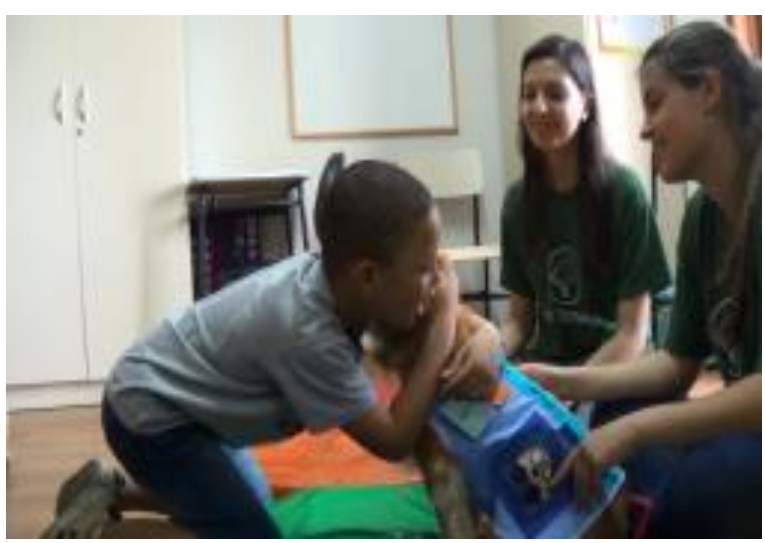

Figura 3: criança que apresenta TEA na TAA.

\section{Conclusão}

Dentro deste contexto, constata-se que a presença do animal contribui para uma imediata interação à TAA, entretanto os animais são considerados importante aspecto de motivação na psicoterapia, ou seja, a ponte com o terapeuta e assim este poder alcançar mais rapidamente a criança, possibilitando a modulação da ansiedade e a abertura da possibilidade de vinculação entre paciente e terapeuta.

A TAA com crianças que apresentam o TEA não parece causar estresse aos cães, não interferindo diretamente no bem-estar e na saúde, Desse modo, embora de forma subjetiva, pode-se dizer que as intervenções realizadas não geraram malefícios ao comportamento e ao bem-estar dos animais.

Concluindo, essa abordagem terapêutica promove a socialização e afetividade através do contato direto entre criança-animal; facilita o desenvolvimento de vínculos e estimula a interação social, de forma que com o passar do 
tempo se estabeleça aquisições funcionais significativas. Pode-se afirmar que os cães terapeutas contribuíram muito para este estudo sem nenhum prejuízo ao seu bem-estar.

\section{Referências}

American Psichiatric Association (2014). DSM-5 Manual diagnóstico e estatístico de transtornos mentais, $5^{\mathrm{a}}$ ed. Porto Alegre: Artmed.

Capote, P. S. O., Costa, M. P. R. (2011). Terapia assistida por animais: aplicação no desenvolvimento psicomotor da criança com deficiência intelectual .São Carlos: EDUFSCAR.

Cheline, M. O. M. (2016). Cães, cavalos... E os outros? In: Chelini, M.O.M., Otta. E. (coord). Terapia assistidapor animais. São Paulo: Manole, p. 313-326.

Dotti, J. (2005). Terapia e Animais. 1. ed. São Paulo: Noética.

Eggiman, J. (2006) Cognitive-behavioral therapy: A case report - Animal- Assisted Therapy.Topics in Advanced Practice Nursing e Journal.v. 6 (3). Disponível em: www.medscape.com/viewarticle/545439.

Friesen, L (2010). Exploring Animal-Assisted Program swith Children in School and Therapeutic Contexts. Early Childhood Education Journal , 2010,37 (4): 261-267.

Muñoz, P. O. L., Roma, R. P. S. (2016).Terapia assistida por animais e autismo. In: CHELINI, M.O.M., OTTA. E. (coord). Terapia assistida por animais. São Paulo: Manole, p. 275-287.

Nobre, M. O. N.,Krug, F. D. M., Capella, S. O., Ribeiro, V. P. R, Nogueira, M. T. D., Canielles, C., Tilmann, M. (2017). Projeto pet terapia: intervenções assistidas por animais: uma prática para o benefício da saúde e educação humana. Expressa Extensão. v.22, n.1, p. 11-23, JAN-JUN, http://dx.doi.org/10.15210/ee.v22i1

Rosa, P.D.E.; Rainho, M.R.G, Pereira, G. G. (2015). Revisão sobre ética e bem-estar nas intervenções assistidas por cães. Clínica Veterinária, Ano XX, n. 116, maio/junho; 40-46. 